\title{
Factors affecting functional recovery after surgery and hand therapy in patients with Dupuytrens disease
}

Christina Engstrand, Barbro Krevers and Joanna Kvist

\section{Linköping University Post Print}

\section{Tweet}

N.B.: When citing this work, cite the original article.

Original Publication:

Christina Engstrand, Barbro Krevers and Joanna Kvist, Factors affecting functional recovery after surgery and hand therapy in patients with Dupuytrens disease, 2015, Journal of Hand Therapy, (28), 3, 255-260.

http://dx.doi.org/10.1016/j.jht.2014.11.006

Copyright: Elsevier

http://www.elsevier.com/

Postprint available at: Linköping University Electronic Press

http://urn.kb.se/resolve?urn=urn:nbn:se:liu:diva-121325 
Title:

Factors affecting functional recovery after surgery and hand therapy in patients with Dupuytren's disease.

\section{Authors:}

Christina Engstrand, BSc ROT, PhD student ${ }^{1,2}$

${ }^{1}$ Department of Hand Surgery, Plastic Surgery and Burns, County Council of Östergötland, Rehabilitation Unit, floor 09, 58185 Linköping, Sweden

${ }^{2}$ Division of Physiotherapy, Department of Medical and Health Sciences

Linköping University, 58183 Linköping, Sweden

Barbro Krevers, ROT, PhD

Division of Health Care Analysis, Department of Medical and Health Sciences

Linköping University, 58183 Linköping, Sweden

barbro.krevers@liu.se

Joanna Kvist, RPT, PhD, Associate Professor

Division of Physiotherapy, Department of Medical and Health Sciences

Linköping University, 58183 Linköping, Sweden

joanna.kvist@liu.se

\section{Corresponding author:}

Christina Engstrand

Department of Hand Surgery, Plastic Surgery and Burns

County Council of Östergötland

University Hospital, Rehabilitation Unit, floor 09 
58185 Linköping, Sweden

Phone: +46101031887

christina.engstrand@ regionostergotland.se

No financial benefits have been provided to the authors and this research has not been presented before in any form. 


\section{Abstract}

\section{Study design}

Prospective cohort study.

\section{Introduction}

The evidence of the relationship between functional recovery and impairment after surgery and hand therapy are inconsistent.

\section{Purpose of the study}

To explore factors that were most related to functional recovery as measured by DASH in patients with Dupuytren's disease.

\section{Methods}

Eighty-one patients undergoing surgery and hand therapy were consecutively recruited. Functional recovery was measured by the Disability of the Arm, Shoulder and Hand (DASH) questionnaire. Explanatory variables: range of motion of the finger joints, five questions regarding safety and social issues of hand function, and health-related quality of life (Euroqol).

\section{Results}

The three variables "need to take special precautions", "avoid using the hand in social context", and health-related quality of life (EQ-5D index) explained $62.1 \%$ of the variance in DASH, where the first variable had the greatest relative effect.

\section{Discussion}

Safety and social issues of hand function and quality of life had an evident association with functional recovery.

\section{Keywords}


Dupuytren's contracture, emotional function, range of motion, recovery of function, quality of life

Level of evidence: IV 


\section{Introduction}

Impairment in hand function and restrictions in performance of daily activities are the motives for patients with Dupuytren's disease (DD) to seek medical care. (1) DD is a benign soft tissue disorder affecting the palmar fascia. DD causes impairment in hand function by finger joint contractures in one or several fingers, in one or both hands, with inability to extend the finger joints. Both surgical and non-invasive methods exist for correction of the extension deficit but the treatment does not cure the disease, and the rate of recurrence is high. $(2,3)$ Some surgeons recommend hand therapy after correction of the extension deficit, in order to maintain the gains in finger extension and to restore hand function. $(2,4)$

For patients with DD, the most commonly reported outcome measures are change in extension deficit of the finger joints, and the Disability of the Arm, Shoulder and Hand (DASH) questionnaire. (5) The extension deficit is a direct measure of the impairment whereas DASH is used to measure functional recovery, including impairments, activity limitations and participation restrictions. $(6,7)$ The relationship between these commonly used outcome measures for patients with DD is still unclear; significant but weak correlations has been shown (8) as well as non-significant correlations between extension deficit and DASH score. (9-12) This indicates that the degree of functional recovery is not dependent only on the severity of the contracture. (11)

DD is a disease that mainly affects older men. (13) Although age has not been shown to be significantly correlated with DASH (10), sex has (14-16) with higher DASH scores (worse disability) among women. (16) Furthermore, DD leads to consequences such as activity limitations, safety and social issues of hand function and quality of life issues. $(1,17,18,19)$ Higher DASH score has also shown to correlate with worse general health (20), but the association between DASH and quality of life outcomes after fasciectomy for DD has not been investigated previously. 
Previous research has highlighted the complexity of functional recovery and the need for further research on factors that influence it. $(15,21)$ One aspect that is often overlooked in patient reported outcomes, but identified as important to patients with hand injuries, are emotional functions. (7) For patients with DD, it could be assumed that improving the extension deficit would affect functional recovery measured with DASH. Yet, based on the weak and inconsistent evidence of this relationship, it seems likely that other factors may contribute to the change in how patients rate functional recovery after surgery and hand therapy.

\section{Purpose of the Study}

To explore factors that were most related to functional recovery as measured by DASH in patients with Dupuytren's disease.

\section{Methods}

\subsection{Study design}

Longitudinal data were analyzed from a prospective cohort study that conducted measurements before treatment, and three months after surgery and hand therapy for patients with DD.

\subsection{Participants}

The study participants were recruited from a cohort study of hand function and quality of life among patients with DD, treated in one hand according a standard protocol with fasciectomy and postoperative hand therapy including active exercises and orthotic intervention. (19) The patients were invited to the study in the order they were scheduled for surgery thus forming a consecutive sample. Inclusion criteria for the cohort study were having DD in one or several 
fingers of digit II-V, with an extension deficit of $60^{\circ}$ or more in an isolated finger joint (metacarpophalangeal (MCP) or proximal/distal interphalangeal (PIP and DIP) joint only) or throughout a whole finger (MCP, PIP, DIP together). Additional inclusion criteria for the present study was to have complete DASH questionnaires from before and three months after surgery. Exclusion criteria were having treatment for another upper extremity disease or not speaking X. Of 123 available patients, 24 did not meet the inclusion criteria for the present study, and 10 declined to participate. For four patients the measurements of range of motion were incomplete and four patients dropped out at the three-month follow-up. Finally, 81 patients, 71 men and 10 women, with a mean age of $68 \pm 8$ years were included in the present study. Table 1 summarizes the details of the study sample and type of intervention.

The Regional Ethical Review Board in Linköping, Sweden approved the study and all participants gave informed consent to participate in the study.

\subsection{Procedures}

Measurements were carried out before treatment (on the day of surgery) and three months after surgery and hand therapy. Two occupational therapists (OT) that were not involved in the postoperative hand therapy performed the measurements before treatment and three months after surgery. The same OT followed each patient, except for five patients living further away from the hospital, where local OTs specially trained for the study performed the follow-up measurements.

\subsection{Measurements}

Functional recovery was measured by the Swedish version of the DASH questionnaire. The 30 items are rated on a five-point scale ( $1=$ no difficulty, $5=$ unable), giving a total DASH score ranging from " $0=$ no disability" to " $100=$ severest disability". The DASH was created as an outcome measure for different upper extremity disorders and is a reliable and valid 
instrument for patients with disorders affecting the arm, wrist and hand. $(20,22)$ The items in DASH have been analyzed in relation to the International Classification on Functioning, Disability and Health (ICF), and 27 of 30 questions in the disability/symptoms scale have been stated to cover the domains of body function, activity and participation. (6) Normative values based on a general American population have been reported with an average of 10.1 (SD 14.68) in DASH score. (23) A change of 15 score points in DASH is considered as a clinical important change in patients with shoulder, wrist and hand disorders. (24)

Range of motion (ROM): Range of motion in the present study refers to total active extension deficit and total active flexion of the finger joints only. Extension and flexion of isolated finger joints in the affected finger were measured with a goniometer according to guidelines. (25) The total active extension deficit was calculated as the sum of the maximum active extension deficit in the MCP, PIP and DIP joint. If hyperextension was present in the DIP joint, it was stated as 0 degrees in the analysis to avoid an underestimation of the total active extension deficit. The total active finger flexion was calculated as the sum of the maximum active flexion in the MCP, PIP and DIP joint. For patients with two or three affected fingers, an average of range of motion outcomes was calculated. (26)

Safety and social issues of hand function were investigated using five questions (19):

1. Do you worry about not being able to trust in your hand function?

2. For security reasons, do you need to take special precautions due to your hand function?

3. Are you afraid of hurting your hand?

4. Are you concerned about the appearance of your hand?

5. Do you avoid using your hand in social contexts? 
Response options ranged from " $1=$ to a large degree" to " $10=$ not at all". These five questions were developed by the authors in order to capture emotional functions (27) and were based on clinical experience and theoretical knowledge. In order to achieve face and content validity of the five questions a consensus discussion was carried out with experts and clinicians with experience of patients with DD.

Health-related quality of life consisted of the Swedish version of Euroqol (EQ-5D), with two parts: the EQ-5D index is based on valuation of health state in five domains in relation to quality of life, and the index ranges from $-0.594=$ "worst imaginable health state" to $1=$ "full health". EQ VAS is a vertical visual analogue scale for self-reported health, and the scale ranges from $0=$ "worst imaginable health state" to $100=$ "best imaginable health state". (28)

\subsection{Data analysis}

The analysis was based on longitudinal data i.e. change scores from before treatment to three months after surgery and hand therapy. Descriptive statistics were applied to show the characteristics of the study population. The extent of the disease was represented by the variables having one or multiple (two or three) affected fingers, and unilateral versus bilateral disease. Dichotomous variables (sex, one/multiple affected fingers, unilateral/bilateral disease) were explored with the Student's t-test to investigate if there were any differences in functional recovery. Associations between functional recovery measured with DASH and the other outcome variables were explored with bivariate and multivariable analyses. Bivariate analysis involved the Pearson's correlation coefficient for interval scales (ROM, DASH, EQ5D index, EQ VAS) and Spearman's rank correlation coefficient for ordinal/categorical scales. From the bivariate analysis, variables with an alpha level of $<0.1$ were chosen to create a regression model that best explained functional recovery measured with DASH. We applied a multivariable regression analysis, of the linear backwards type, with an alpha level 
of 0.06 as a limit for removal of variables, with DASH change score as the dependent variable. Nine independent variables were included in the initial regression analysis: total active extension deficit, total active flexion, worry about not trusting in hand function, need to take special precautions due to hand function, fear of hurting the hand, avoid using the hand in social context, EQ VAS, EQ-5D index and sex. Alpha level was set to 0.05 for significance. In the EQ-5 D index, 0.1 point represented one scale step of change in the regression analysis.

Based on the observed model fit $\left(r^{2}=0.6\right)$, the nine predictors entered into the regression analysis, and the sample size of 68 , the power of the study exceeded $90 \%$. The SPSS version 22.0 was used for data management and analysis.

\section{Results}

Data on the outcome measures from baseline data (before treatment) and three months followup is summarized in table 2 . There was no difference in functional recovery between patients with one or multiple affected fingers $(\mathrm{p}=0.863)$ or patients with unilateral versus bilateral DD $(\mathrm{p}=0.345)$. However, there was a difference in functional recovery between men and women, with a greater improvement in DASH score among women (mean change 18, SD 18) compared to men (mean change $8, \mathrm{SD} 13)(\mathrm{p}=0.036)$. Improvement of functional recovery measured by DASH was positively associated with improvement of total active extension deficit $(r=0.256)$, total active finger flexion $(r=0.302)$, four out of five questions regarding safety and social issues of hand function $(r \leq 0.700)$, and quality of life $(r \leq 525)$ (table 3$)$. As age and "concerns about the appearance of the hand" were not associated with functional recovery (table 3), these variables were not included in the multivariable regression analysis. 
Six of the nine variables entered into the multivariable regression analysis were removed stepwise based on their high $\mathrm{p}$-value $(\mathrm{p}>0.06)$ during the backwards procedure. The six variables excluded were total active extension deficit, total active finger flexion, "worry about not trusting in hand function", "fear of hurting the hand", EQ VAS and sex. The final model best explaining the variation in the DASH included three variables; "need to take special precautions due to hand function", "avoid using the hand in social context", and health-related quality of life measured with the EQ-5D index (table 4). The standardized coefficients $(\beta)$ showed that an improvement in "need to take special precautions due to hand function" had the greatest relative effect of the individual variables in the final model. The unstandardized coefficient (B) for this variable showed that an improvement of one score point in this variable was associated with an improvement in DASH with 2.55 score points. The overall model fit showed that improvements in the three variables in the final model explained $62.1 \%$ of the variance in DASH score.

\section{Discussion}

This study showed that improvement in functional recovery was positively associated with improvement in ROM, four of five questions regarding safety and social issues and healthrelated quality of life. These variables contributed individually but with different strengths, to the improvement in DASH score. The improvement in functional recovery measured with the DASH score was to a considerable extent explained by safety and social issues and healthrelated quality of life.

For patients with DD, the inability to extend the finger joints represents the impairment caused by the disease. In the present study, there was a small but significant, positive association between reduced total active extension deficit and improvement in the DASH. These findings were in line with previous research on associations between ROM and the 
DASH before surgery. (8) Nevertheless, the total active extension deficit was a less important factor for functional recovery when it interacted with other factors in the regression model, and it was not part of the final model best explaining the functional recovery. In the present study, functional recovery differed between men and women and the finding of greater functional recovery among women points in the same direction as previous research. (16) But similar to the total active extension deficit, sex was a less important factor for functional recovery when put into a regression analysis with other factors and was not part of the final model.

Although interventions in hand surgery and therapy often target physical function, the overall goal is to enhance the individual's ability to execute tasks and to participate fully in life situations. (29) From the patients' perspective, performance of daily activities, interaction with others or quality of life may be more important outcomes than the degree of impairment. (17) Therefore, we assumed that change in factors other than total active extension deficit would contribute to the patients' rating of functional recovery with DASH. The present study confirmed this assumption as safety and social issues and quality of life were significantly associated with the improvement in DASH. Based on the ICF (27), the two questions regarding safety and social issues in the final multivariable regression model, can be considered as emotional functions. Emotional functions, such as anxiety about the ability to cope with daily life or to handle occupational demands, have previously been identified as important to patients with hand injuries/disorders but are not captured in patient reported outcomes such as the DASH. (7) In the DASH there are two questions which target capacity or self-confidence in a more general way, (item 22, "During the past week, to what extent has your arm, shoulder or hand problem interfered with your normal social activities with family, friends, neighbors or groups?” and item 30, “I feel less capable, less confident or less useful because of my arm, shoulder or hand problem"). Otherwise, the DASH covers no questions 
about social and safety issues of hand function. The two questions "need to take special precautions due to hand function" and "avoid using the hand in a social context" also touch on the themes found in previous research about the patients' perspective on DD. $(1,17)$ The third variable in the final regression model was quality of life measured with the EQ-5D index. A higher DASH score has previously been shown to correlate with depression $(14-16,21)$ and since one of the dimensions captured in the EQ-5D index is anxiety/depression, this might have contributed to the findings in the present study.

Functional recovery is a complex phenomenon, which is based on interactions that are specific and not always predictable. (27) However, using a method based on the assumption of linearity, such as multivariable regression analysis, can be helpful in capturing patterns or regularities that might be present in the complex phenomenon that is studied. Although conclusions cannot be drawn about the impact of individual variables on functional recovery, the multivariable regression can help us find out more about the underlying aspects. In the present study we choose to use the follow up three months after surgery and hand therapy, which represent a common time point for clinical evaluation of the short-term outcome.

\section{1 Limitations}

The few women in the study cause a limitation in the statistical analysis therefore further research is needed to examine if sex has influence on functional recovery or not. Yet the ratio between men and women in the present study reflect the epidemiology and was in line with previously reported data. (30) The rule of thumb of having a sample size of at least ten cases per variable in the regression analysis (31) was not reached in the initial phase of the analysis. Thus we used a backwards type of regression analysis where the variables were removed based on the alpha level, which was a proper way to reduce the number of variables. In the final model there were three variables left, i.e. giving a sample size of 22 cases per variable. 
Further, there might also be other variables beyond the nine variables entered in the multivariable regression analysis in the present study that are important for functional recovery.

\subsection{Conclusion}

The study showed that emotional functions and quality of life had an evident association with functional recovery after surgery and hand therapy. Thus, these factors are important supplements to physical measurements to consider when evaluating interventions for patients with DD. 


\section{Acknowledgement}

We thank the Department of Hand surgery, Plastic surgery and Burns, University Hospital,

Linkoping, Sweden for providing practical support and Henrik Magnusson for statistical

support. The study was supported by grants from the Medical Research Council of southeast

Sweden (FORSS-72231) and the County Council of Ostergotland LIO-77311, Sweden.

\section{Conflicts of interests}

There are no conflicts of interest 


\section{References}

1. Pratt A L, Byrne G. The lived experience of Dupuytren's disease of the hand. J Clin Nurs. 2009;12:1793-1802.

2. Shih B, Bayat A. Scientific understanding and clinical management of Dupuytren's disease. Nat Rev Rheumatol. 2010;6:715-726.

3. Hindocha S, Stanley J K, Watson S, Bayat A Dupuytrens diathesis revised: evaluation of prognostic indicators for risk of disease recurrence. J Hand Surg Am. 2006;31A:1626-1634.

4. Dahlin L B, Bainbridge C, Szczypa P P, Cappelleri J C, Guerin D, Gerber R A. Current trends in the surgical management of Dupuytren's disease in Europe: the surgeon's perspective. European orthopaedics and traumatology. 2012;1:25-30.

5. Ball C, Pratt A L, Nanchahal J. Optimal functional outcome measures for assessing treatment for Dupuytren's disease: a systematic review and recommendations for future practice. BMC musculoskeletal disorders. 2013;14:131.

6. Dixon D, Johnston M, McQueen M, Court-Brown C. The Disabilities of the Arm, Shoulder and Hand Questionnaire (DASH) can measure the impairment, activity limitations and participation restriction constructs from the International Classification of functioning, Disability and Health (ICF). BMC Musculoskeletal Disorders. 2008;9:114 DOI: 10.1186/1471-2474-9-114.

7. Coenen M, Kus S, Rudolf K D, Muller G, Berno S, Dereskewitz C, et al. Do patientreported outcome measures capture functioning aspects and environmental factors important to individuals with injuries or disorders of the hand? J Hand Ther. $2013 ; 4: 332-342$.

8. Jerosch-Herold C, Shepstone L, Chojnowski A, Larson D. Severity of contracture and self-reported disability in patients with Dupuytren's contracture referred for surgery. J Hand Ther. 2011;1:6-10.

9. Engstrand C, Boren L, Liedberg G M. Evaluation of activity limitation and digital extension in Dupuytren's contracture three months after fasciectomy and hand therapy interventions. J Hand Ther. 2009;22:21-26.

10. Zyluk A, Jagielski W. The effect of the severity of the Dupuytren's contracture on the function of the hand before and after surgery. J Hand Surg Eur. 2007;3:326-329. 
11. Degreef I, Vererfve P B, De Smet L. Effect of severity of Dupuytren's contracture on disability. Scand J Plast Reconstr Surg Hand Surg. 2009;1:41-42.

12. Mohan A, Vadher J, Ismail H, Warwick D. The Southampton Dupuytren's Scoring Scheme. Journal of plastic surgery and hand surgery. 2014;1:28-33.

13. Hindocha S, McGrouther D A, Bayat A. Epidemiological evaluation of Dupuytren's disease incidence and prevalence rates in relation to etiology. Hand. 2009;3:256-269.

14. Niekel M C, Lindenhovius A L, Watson J B, Vranceanu A M, Ring D. Correlation of DASH and QuickDASH with measures of psychological distress. J Hand Surg Am. 2009;8:1499-1505.

15. Calderon S A, Zurakowski D, Davis J S, Ring D. Quantitative Adjustment of the Influence of Depression on the Disabilities of the Arm, Shoulder, and Hand (DASH) Questionnaire. Hand. 2010;1:49-55.

16. Ring D, Kadzielski J, Fabian L, Zurakowski D, Malhotra L R, Jupiter J B Selfreported upper extremity health status correlates with depression. J Bone Joint Surg Am. 2006;9:1983-1988.

17. Wilburn J, McKenna S P, Perry-Hinsley D, Bayat A. The impact of Dupuytren's disease on patient activity and quality of life. J Hand Surg Am. 2013;6:1209-1214.

18. Thoma A, Kaur M, Ignacy T A, Levis C, Martin S, Duku E, et al. Health Related Quality of Life in Patients undergoing Palmar Fasciectomy for Dupuytren's Disease. Plastic and reconstructive surgery. 2014;E-pub ahead of print.

19. Engstrand C, Krevers B, Nylander G, Kvist J. Hand function and quality of life before and after fasciectomy for Dupuytren's disease. J Hand Surg Am. 2014;7:1333-1343.

20. Atroshi I, Gummesson C, Andersson B, Dahlgren E, Johansson A. The disabilities of the arm, shoulder and hand (DASH) outcome questionnaire: reliability and validity of the Swedish version evaluated in 176 patients. Acta Orthop Scand. 2000;6:613-618.

21. Vranceanu A M, Jupiter J B, Mudgal C S, Ring D. Predictors of pain intensity and disability after minor hand surgery. J Hand Surg Am. 2010;6:956-960.

22. Gummesson C, Atroshi I, Ekdahl C. The disabilities of the arm, shoulder and hand (DASH) outcome questionnaire: longitudinal construct validity and measuring selfrated health change after surgery. BMC Musculoskelet Disord. 2003;4:11. 
23. Hunsaker F G, Cioffi D A, Amadio P C, Wright J G, Caughlin B. The American academy of orthopaedic surgeons outcomes instruments: normative values from the general population. J Bone Joint Surg Am. 2002;2:208-215.

24. Beaton D E, Katz J N, Fossel A H, Wright J G, Tarasuk V, Bombardier C. Measuring the whole or the parts? Validity, reliability, and responsiveness of the Disabilities of the Arm, Shoulder and Hand outcome measure in different regions of the upper extremity. J Hand Ther. 2001;2:128-146.

25. Engstrand C, Krevers B, Kvist J. Interrater Reliability in Finger Joint Goniometer Measurement in Dupuytren's Disease. Am J Occ Ther. 2012;1:98-103.

26. Altman D G, Bland J M. Statistics notes. Units of analysis. BMJ. 1997;7098:1874.

27. WHO. ICF - International classification of functioning, disability and health. Geneve: World Health Organization; 2001.

28. Oemar M, Oppe M. EQ-5D-3L user guide. Basic information on how to use the EQ5D-3L instrument. Rotterdam: EuroQol Group; 2013.

29. Dimick M P, Caro C M, Kasch M C, Muenzen P M, Fullenwider L, Taylor P A, et al. 2008 practice analysis study of hand therapy. J Hand Ther. 2009;4:361-375.

30. Wilbrand S, Ekbom A, Gerdin B. The sex ratio and rate of reoperation for Dupuytren's contracture in men and women. J Hand Surg Eur. 1999;4:456-459.

31. Petrie A, Sabin C. Medical statistics at a glance. 3 ed. West Sussex: Wiley-Blackwell; 2009. 
Table 1. Patient characteristics of the study cohort $n=81$ and given intervention, presented in proportions (\%).

\begin{tabular}{|c|c|c|}
\hline \multicolumn{3}{|l|}{ Variables } \\
\hline & $n$ & $\%$ \\
\hline \multicolumn{3}{|l|}{ Sex } \\
\hline Male & 71 & 88 \\
\hline Female & 10 & 12 \\
\hline \multicolumn{3}{|l|}{ Diabetes } \\
\hline Yes & 9 & 11 \\
\hline No & 72 & 89 \\
\hline \multicolumn{3}{|l|}{ Heredity of $D D$} \\
\hline Yes & 38 & 47 \\
\hline No & 16 & 20 \\
\hline Unknown & 27 & 33 \\
\hline \multicolumn{3}{|l|}{ Disease duration } \\
\hline $0-5$ years & 20 & 25 \\
\hline $6-10$ years & 30 & 37 \\
\hline $11-15$ years & 13 & 16 \\
\hline$>15$ years & 18 & 22 \\
\hline \multicolumn{3}{|c|}{ Number of affected fingers } \\
\hline One finger & 64 & 79 \\
\hline Two fingers & 13 & 16 \\
\hline Three fingers & 4 & 5 \\
\hline \multicolumn{3}{|l|}{ DD bilateral } \\
\hline yes & 58 & 72 \\
\hline no & 23 & 28 \\
\hline \multicolumn{3}{|l|}{ Treated hand } \\
\hline right & 43 & 53 \\
\hline left & 38 & 47 \\
\hline \multicolumn{3}{|c|}{ Operated fingers $(n=102)$} \\
\hline Index & 1 & 1 \\
\hline Middle & 8 & 8 \\
\hline Ring & 28 & 27 \\
\hline Little & 65 & 64 \\
\hline \multicolumn{3}{|l|}{ Type of surgery } \\
\hline Fasciectomy & 64 & 79 \\
\hline + open palm* & 8 & 10 \\
\hline+ volar release $^{\dagger}$ & 9 & 11 \\
\hline \multicolumn{3}{|c|}{ Complications during surgery } \\
\hline Nerve injury & 3 & 4 \\
\hline Blood vessel injury & 0 & 0 \\
\hline \multicolumn{3}{|c|}{ Complications after surgery } \\
\hline CRPS & 4 & 5 \\
\hline Infection & 6 & 7 \\
\hline
\end{tabular}

*Open palm technique was used if there was a shortage of skin or bad skin quality. 
${ }^{\dagger}$ The volar plate of the PIP joint was removed if there was a residual extension deficit of 25$30^{\circ}$ in the PIP joint after the fasciectomy.

Table 2. Physical measurement and self-reported outcomes before surgery and at three months follow up, presented as mean (m), standard deviation (SD) or median (md), and interquartile range (Iqr).

\begin{tabular}{|c|c|c|c|c|c|}
\hline \multirow[t]{2}{*}{ Variables } & & \multicolumn{2}{|c|}{ Before surgery } & \multicolumn{2}{|c|}{3 months } \\
\hline & & $\mathrm{n}$ & $\mathrm{m} \pm \mathrm{SD}$ & $\mathrm{n}$ & $\mathrm{m}=\mathrm{SD}$ \\
\hline Functional recovery & DASH score* & 81 & $21 \pm 14$ & 81 & $12 \pm 13$ \\
\hline \multirow[t]{2}{*}{ Range of motion } & Total active extension deficit in degrees ${ }^{\dagger}$ & 81 & $109 \pm 32$ & 81 & $33 \pm 19$ \\
\hline & Total active finger flexion in degrees $t$ & 81 & $239 \pm 16$ & 81 & $222 \pm 26$ \\
\hline \multirow[t]{3}{*}{ Quality of life } & EQ- VAS 5 & 79 & $80 \pm 15$ & 76 & $79 \pm 17$ \\
\hline & EQ-5D index & 79 & $0.82 \pm 0.13$ & 79 & $0.86 \pm 0.17$ \\
\hline & & $\mathrm{n}$ & md (Iqr) & $\mathrm{n}$ & md (Iqr) \\
\hline Safery \& social issues & 1. Worry about not trusting in hand function & 79 & $5(3-7)$ & 79 & $8(6-10)$ \\
\hline \multirow[t]{4}{*}{ of hand function } & 2. Need to take special precautions due to hand function & 78 & $6(4-8)$ & 79 & $8(6-10)$ \\
\hline & 3. Fear of hurting the hand & 80 & $5(4-8)$ & 78 & $9(7-10)$ \\
\hline & 4. Concemed about appearance of the hand & 80 & $8(5-10)$ & 79 & $10(10-10)$ \\
\hline & 5. Avoid using the hand in social context & 80 & $9(5-10)$ & 79 & $10(10-10)$ \\
\hline
\end{tabular}


Table 3. Associations between functional recovery (improvement on DASH from before surgery to three months) and other variables. The data is presented as Pearson's (r) or Spearman's (rho).

\begin{tabular}{lcccc}
\hline Variables & $\mathbf{n}$ & $\mathbf{r}$ & $\mathbf{r h o}$ & $\mathbf{p}$ \\
\hline Age & 81 & .085 & .449 \\
\hline Total active extension deficit improved & 81 & .256 & $\mathbf{. 0 2 1}$ \\
\hline Total active flexion improved & 81 & .302 & $\mathbf{. 0 0 6}$ \\
\hline Worry about hand function improved & 77 & .693 & $\mathbf{. 0 0 0}$ \\
\hline Need to take special precautions due to hand function improved & 76 & .700 & $\mathbf{. 0 0 0}$ \\
\hline Fear of hurting the hand improved & 78 & .497 & $\mathbf{. 0 0 0}$ \\
\hline Concerned about the appearance of the hand improved & 78 & .110 & .337 \\
\hline Avoid using the hand in social context & 78 & .283 & $\mathbf{. 0 1 2}$ \\
\hline EQ VAS improved & 74 & .275 & $\mathbf{. 0 1 8}$ \\
\hline EQ-5D index improved & 77 & .525 & $\mathbf{. 0 0 0}$
\end{tabular}


Table 4. Analysis of independent variables best explaining functional recovery measured with DASH. Data for the final model presented as the unstandardized coefficient (B) with $95 \%$ confidence intervals, the standardized coefficient $(\beta)$ for the independent variables, and adjusted coefficient of determination $\left(\operatorname{adj} \mathrm{r}^{2}\right)$ for overall model fit.

\section{Multivariable regression analysis}

n $\quad$ B $\quad 95 \%$ CI for B $\quad \beta \quad$ p-value $\operatorname{adj~} r^{2}$

\begin{tabular}{lll}
\hline Independent variables & Lowe & Uppe \\
& $\mathrm{r}$ & $\mathrm{r}$
\end{tabular}

$\begin{array}{llllllll}\text { Need to take special precautions } & 68 & 2.55 & 1.85 & 3.24 & .577 & \mathbf{. 0 0 0}\end{array}$

due to hand function improved

$\begin{array}{llllllll}\text { Avoid using the hand in social } & & 68 & 0.73 & 0.002 & 1.45 & .156 & \mathbf{. 0 4 9}\end{array}$

context improved

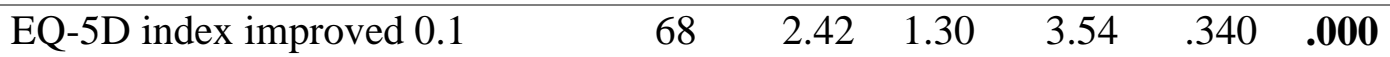

